Relations industrielles

Industrial Relations

\title{
Notions essentielles de comptabilité de gestion, Tome I et II, par Pierre Maingaud, Sirey, Paris, 1964. 265 pages (tome I) 279 (tome II).
}

\section{Bertrand Belzile}

Volume 20, numéro 1, 1965

URI : https://id.erudit.org/iderudit/027556ar

DOI : https://doi.org/10.7202/027556ar

Aller au sommaire du numéro

Éditeur(s)

Département des relations industrielles de l'Université Laval

ISSN

0034-379X (imprimé)

1703-8138 (numérique)

Découvrir la revue

Citer ce compte rendu

Belzile, B. (1965). Compte rendu de [Notions essentielles de comptabilité de gestion, Tome I et II, par Pierre Maingaud, Sirey, Paris, 1964. 265 pages (tome I) 279 (tome II).] Relations industrielles / Industrial Relations, 20(1), 196-197. https://doi.org/10.7202/027556ar

Tous droits réservés @ C Département des relations industrielles de l'Universite Laval, 1965
Ce document est protégé par la loi sur le droit d'auteur. L'utilisation des services d'Érudit (y compris la reproduction) est assujettie à sa politique d'utilisation que vous pouvez consulter en ligne.

https://apropos.erudit.org/fr/usagers/politique-dutilisation/ 
torique. Ils étudient trois des attitudes les plus marquantes; l'attitude libérale, l'attitude marxiste et l'attitude keynesienne.

En matière d'emploi le premier problème que doit se poser toute collectivité humaine, est d'équilibrer l'offre totale d'emplois émanont de l'économie, et la demande totale d'emplois émanant de la population. La façon de résoudre le problème de l'emploi dépend des options économiques et politiques du système considéré. Lo théorie libérale considère que la condition nécessaire et suffisante pour éliminer tout chômoge durable est de maintenir un marché du travail libre et concurrentiel, mais pour y arriver on doit faire interverir l'Etat, (bureaux de placements, aide aux chômeurs), et cela même si les thèses libérales ont longtemps favorisé le laisser-faire. Les auteurs croient que les thérapeutiques libéroles s'ovèrent radicalement inefficoces; ils démentissent l'optimisme libéral por l'expérience historique

Les auteurs exposent ensuite la critique morxiste à l'égard du système capitaliste. Partie d'un coractère tout à fait théorique, et cela se comprend. Des renseignements concrets sur la mise en oeurre effective des politiques de l'emploi en poys socialistes, oinsi que les résultats de ces politiques. demeurent particulièrement difficiles à obtenir. Quant d̀ la réponse donnée par le néo-capitalisme, les auteurs en présentent les trois instruments théoriques sur lesquels se fonde la politique du plein emploi, stimulation de l'investissement privé en occroissont sa rentabilité, stimulation de la consommations par lo redistribution des revenus, finoncement des dépenses publiques supplémentoires par le déficit budgétaire.

Les auteurs constotent que le problème d'emploi ne sera pas résolu en établissant un équilibre global et approximatif entre les emplois offerts et les emplois acceptés. II faudro étudier les problèmes particuliers à l'emploi qui, sous le titre la Structure de L'Emploi, sont l'objet de la seconde partie du volume. Par une onalyse statistique les outeurs répondent oux questions suivontes; qui travaille en France? Combien de temps travaille-t-on? De quelle façon se modifie sans cesse le tableau ou le carte du travail? Et enfin que fait-on pour préparer les hommes aux travaux de demain?

Les résultats fournissent les éléments nécessaires pour envisager dans la dernière parties, Contraintes et Options, la politique française de l'emploi, et particulièrement lo planification de l'emploi. Il est assez difficile de parler de réelle planification de l'em- ploi en France. II s'agirait surtout d'une prise en considérotion des problèmes de l'empioi dans l'éraboration générale du Plan. Il serait intéressant de faire une étude comparative de la situation de la planification de l'emploi en France et de celle du Québec, qui ò dire vrai est encore un ensemble de mesures fragmentaires. bien dispersées. Une adaptation du Plan français serait peut-être souhoitoble, non pas une imitation.

Les outeurs terminent leur volume dans des considérations généroles sur l'emploi et le progrès de la civilisation; selon eux l'ovenir de la civilisation semble lié très intimernent à lo façon dont on s'y prendro pour accroitre les forces productives de I'humanité, et pour répartir plus équitablement non seulement les produits de l'activité humaine, mais aussi les pouvoirs directeurs de cette activité.

La lecture de cet ourrage s'avère utile à ceux qui souhaitent s'informer des problèmes actuels de l'emplois. A ceux qui voudraient poursuivre l'étude des questions traitées, le volume offre plus de dix pages de références biblingraphiques très précieuses.

\section{Jean COSSETTE}

\section{Notions essentielles de comptabillité de ges-}

tion, Tome 1 et 11, par Pierre Maingaud Sirey, Paris, 1964. 265 pages (tome 1) 279 pages (tome II)

Voici les deux premiers de trois ourrages de $M$. Maingaud, qui doivent couvrir le programme des trois années des Ecoles supérieures de commerce, en France. Nous sommes d'abord heureux de constater que l'auteur présente la comptabilité bien plus comme un outil de gestion que comme un répertoire de techniques d'enresgistrement des transactions.

Pendant trop longtemps, la comptabilité o été enseignée en vase clos, en quelque sorte pour ele-même. De plus en plus au contraire, elle constitue l'une des disciplines fondamentales de l'administration de l'en. treprise et partant les techniques et analyses comptables doivent être établies en fonction des problèmes majeurs de l'entreprise moderne, au sens large du terme. En un mot, la déspécialisation et le décloissonnement sont des impératifs là comme ailleurs. Le comptable ne doit plus travailler seul et pour lui-même, mais avec d'autres spécialistes et en vue d'éclairer le mieux possible les décisions des dirigeants de l'entreprise. 
Dans le tome 1, l'outeur décrit brièvement le rôle et le fonctionnement de l'entreprise de même que lo fonction comptable dans l'entreprise. Puis vient l'explicotion des méconismes comptables qui s'étend sur une trentaine de pages seulement, alors que les volumes traditionnels lui occordent une importance relotive beaucoup plus grande. Por cilleurs, M. Maingaud insiste plus lonquement sur le bilan et l'exploitation. Enfin deux brefs exposés l'un concernant l'inventoire et lo détermination des résultats, et loutre troitant du système dit classique de la technique de bose des enregistrements comptribles.

Dans le tome 11 , an retrouve d'abord une description de l'évolution qu'ont connue les techniques d'enregistrement. Vient ensuite la Dortie la plus importonte et la plus intéressante de cet ouvrage qui traite des ćtudes de rentabilité et la compabilité anoIytique d'exploitation. Tout au long de cette partie, on peut percevoir cette préoccupa. tion de l'auteur, que nous signalions plus haut, d'enseigner la comptabilité en tant qu'outil de gestion. La partie suivante couvre la comptabilité des sociétés commerciales; celle-ci bien entendu est particulière à la législation française et diffère de ce que nous connaissons au Canada et au Québec. Pour finir, on trouve une étude critique des enonciations du bilan.

Le titre de ces deux ourrages « Notions essentielles de comptabilité de gestion " indique donc bien l'effort de l'auteur de rendre la comptabilité davantage utilisable pour fins de gestion. Nous faisons simplement remarquer ici que d'autres auteurs vont encore plus loin dans cette direction. Et finalement, une réserve pour les lecteurs canodiens: la comptabilité française, guidée por le Plan Comptable général, diffère quelque peu, dans sa terminologie et sa présentation, de notre comptabilité canadienne, sans répéter la différence mentionnée au paragraphe précédent.

\section{Bertrand BELZILE}

The Supervision of Personnel, par John $M$. Pfiffner et Marshall Fils, Prentice-Hall Inc., Englewood Cliffs, New Jersey, 3ème édition, 1964, 369 pp.

Les chercheurs en relations humaines ont réussi déjà à accumuler beaucoup d'évidence sur la supériorité de la supervision * démocrotique ». i.e. centrée sur les individus. Dons le même courant d'idée, les auteurs de ce volume partent de l'hypothèse de base qu'il est possible de former une équipe de surveillants très compétents sur le plans des relations interpersonnelles et aussi sur le plan des résultats économiques tels qu'espérés par lo houte-direction. Ceci veut dire, d'abord, que le supérieur doit établir un a type de relations » où lui-même et son subordonné auront l'occosion de développer leur personnolité respective. Ceci nous amène aussi à repenser le rôle du superviseur, le contexte orgonisationnel dons lequel il joue ce rôle, c'est-à-dire la mentolité troditionnelle des dirigeants, les structures de Yorganisation, les attitudes et croyances des subordonnés et les communications etc... De plus, il faut reconsidérer les méthodes traditionnelles et nouvelles de formation des codres.

\section{Le supérieur : « un clinicien »}

Dans le contexte où la supervision devient essentiellement une activité de développement de la personnalité, le surveillant joue le rôle d'une clinicien en incitant le subordonné à modifier ses attitudes et son comportement non pas par la force, la manipulation des sanctions et des récompenses, mais bien par la création d'une atmosphère où le subordonné constate la nécessité d'un changement, tend à acquérir une moturité émotionnelle, une plus grande sociabilité, et une habitude à prendre des responsabilités.

Foce à l'incompatibilté entre les exigences de l'organisation sociale de l'entreprise et le désir de perfectionnement chez l'individu à tous les niveaux occupationnels, le supérieur en qualité de clinicien doit s'efforcer de réconcilier les différences, de réduire les tensions entre les exigences structurelles et les aspirations personnelles.

Face à l'union, le supérieur hiérarchique peut utiliser la méthode clinique tout en ne rejetant pas l'approche légale. Comme clinicien, il doit écouter, conseiller, enregistrer des faits, se placer dans la situation de l'autre, agir dans une atmosphère de conciliation amicale. L'approche légale est déjò connue, il n'est pas nécessaire de la présenter ici.

Commme clinicien, le supérieur ne doit pas perdre de vue l'aspect économique de son rôle, Des considérations de coûts, de profits, de productivité doivent foire aussi l'objet de son attention.

\section{Pour un nouveau \\ a climat organisationnel .}

L'attitude autocratique et conservative de la haute direction constitue un frein à l'ins- 